\title{
MKL1 and STAT3 activate the activity of the luciferase reporter plasmid containing the CAAP1 gene promoter
}

\author{
Jun-Yan $\mathrm{Li}^{1,2 *}$, Zhu $\mathrm{Yu}^{1}$, Feng-Yun Wang ${ }^{1}$. \\ ${ }^{1}$ Henan Vocational College of Applied Technology, 450042, P.R.China. \\ ${ }^{2}$ Institute of Biology and Medicine, Wuhan University of Science and Technology, Wuhan, 430065, PR China.
}

\begin{abstract}
Breast cancer is the leading cause of cancer death in women worldwide. The etiology of the disease is not yet clear. We know that MKL1 and STAT3 play an important part in the development and progression of breast cancer. CAAP1 is a ubiquitous and highly conserved protein that is closely related to the apoptotic process of tumors. However, the definitive transcriptional mechanism of the CAAP1 gene is still unclear. In our study, we constructed a luciferase reporter plasmid for the human CAAP1 gene promoter. Then one or both of the two overexpression vectors of MKL-1 and STAT3 were co-transfected into MCF-7 cells with CAAP1 promoter plasmid, and we then tested activation of the CAAP1 promoter by luciferase reporter assay. The results show that compared with the transfected pcDNA3.1 group, MKL1 can evidently increase the transcription activity of the CAAP1 gene promoter, while the STAT3 group can slightly upregulate the transcription activity of the CAAP1 gene promoter. Our research will further reveal the relationship between CAAP1 and the occurrence and development of breast cancer cells, and provide a new idea and direction for the cures of breast cancer.
\end{abstract}

\section{Introduction}

Breast cancer is the most common malignant tumor that occurs in breast epithelial tissue[1]. Breast cancer has a high mortality rate and has become a common tumor that threatens the physical and mental health of women[2]. The etiology of its onset is not yet clear. Using current diagnostic tools, early detection of breast cancer remains a challenge. At present, more attention is paid to the study of overexpression or abnormal activation of related proteins in the onset, development, and metastasis of breast cancer. CAAP1 (casapase activity and apoptosisihibite 1) is a very conserved and ubiquitously expressed protein[3,4]. It is a potential factor for apoptosis-related signaling by interfering with the activation of caspase-10, while caspase-10 also regulates the production of the caspase-3/9 couple back amplifying cycle to effectively activate the mitochondrial death pathway, which has a huge relationship with the occurrence and development of tumor cells $[5,6]$.

The cardiac protein family and myocardial transcription factor MKL1 are coactivators of serum response factor (SRF)[7]. MKL1 binds to SRF and activates promoter transcription by combining with SRE, also called CARG cassette[8]. Studies have shown that MKL1 is involved in the metastasis and invasion of mammary cancer cells and is a cancer-promoting factor $[9,10]$.

Signal transduction and activator of transcription 3 (STAT3) is widely expressed in a variety of cells and participates in the regulation of physiological functions and pathological processes such as cell growth process, malignant transformation process, and cell apoptosis[11, 12]. Over-activation of STAT3 promotes proliferation, apoptosis and migration of breast cancer cells, and can also lead to malignant transformation of normal breast cells[13-15]. It has been demonstrated that there may be direct protein interactions between MKL1 and STAT3[16,17].

In this study, we successfully amplified the CAAP1 promoter luciferase reporter gene by PCR from the human genome and inserted into a pGL-3 promoter vector. We then co-transfered the CAAP1 promoter plasmid and one or both of the human MKL1 and human STAT3 expression plasmids to human MCF-7 cells, and detected the activation of the CAAP1 promoter using the luciferase reporter gene method. The results show that transfection of each expression vector can promote the transcription activity of CAAP1, and when the two vectors are co-transfected, the activity effect can be improved. Our research will further reveal the molecular mechanisms of the development and progression of breast cancer cells and provide a theoretical basis for the cures of breast tumor.

\section{Materials and Methods}

\subsection{Culture of Cells}

\footnotetext{
* Corresponding author: junyan8067@163.com
} 
Human breast cancer cell line $\mathrm{MCF}-7$ was purchased from ATCC(American Type Culture Collection). MCF-7 cells were cultured in Dulbecco's Modified Eagle's Medium (DMEM) (GIBCO) containing 10\% fetal bovine serum (FBS) at $37^{\circ} \mathrm{C}$ in a $5 \% \mathrm{CO} 2$ incubator.

\subsection{Plasmid Construction}

The human genomic DNA was abstracted from human MCF-7 cell line. PCR amplification of the CAAP1 promoter fragment $(-2428$ to +66$)$ with the following primers: F: 5'ACAGGCTAGCATGTTATAGGACCATCAGG-3'; R: 5'-AGATCTCGAGAGGAAAGTCCGCTGTCTC-3'. The mutant promoter of CAAP1 (binding site with MKL1) primer sequences were as follows: forward: 5'TACAAGGATCGAAGTCAAGTGGGGCCTGGGGG AAGACAGTTTTCCTAGGG-3' and reverse: 5'CCCCACTTGACTTCGATCCTTGTAAGGTAAATCC TGGGGCTGAGCCCACT-3'. The mutant promoter of CAAP1 (binding site with STAT3) primer sequences were as follows: forward: 5'GAGTTCGTAGTCGCTCAGGGGTCTTCAGACTGA GTAGCATCAGTGACCCC-3' and reverse: 5'AAGACCCCTGAGCGACTACGAACTCAGCACAAG AATGCACTTTCTACCTC-3'.

Predenatured at $95^{\circ} \mathrm{C}$ for 5 minutes, denatured at $95^{\circ} \mathrm{C}$ for 1 minute, annealed at $54^{\circ} \mathrm{C}$ for 20 seconds, and extended at $72^{\circ} \mathrm{C}$ for 2 minutes. The PCR reaction was carried out for 32 cycles, run on $1 \%$ agarose gel and stained with ethidium bromide, and finally the PCR product was visualized under UV transmission. The PCR products of CAAP1 and pGL3-promoter vector plasmid were assimilated with restrictive enzymes Nhe I and Xho I for 2 hours at $37^{\circ} \mathrm{C}$. The assimilated CAAP1 fragment and the pGL3-promoter vector were then blended with 4 $\mu \mathrm{L}$ T4 ligase buffer and $2 \mu \mathrm{L}$ T4 DNA ligase, ligated overnight at $16^{\circ} \mathrm{C}$, and it was transformed into E. coli. After 12 hours, single colonies were selected and brought up in LB containing ampicillin. The recombinant plasmid was abstracted and verified..The expression plasmids of STAT3 and MKL1 were vacant with pcDNA3.1; the primers for pcDNA3.1-STAT3 were as follows: forward 5'CAGGATCCTGGCTCAGTGGAACCAGCT-3' and reverse 5'-GACACTCGAGGGTCAATGGTATTGCTG3'; The primers for pcDNA3.1-MKL1 were as follows: forward 5'-CAGGATCC ATGACTCTACTGGAACCTG-3' and reverse 5'ACACTCGAGCTACAAGCAGGAATCCCAG-3'.

\subsection{Transfection}

MCF-7 cells were inoculated in 24-well plates at a density with $1 \times 10^{5}$ cells/well. The cells were transfected with $0.2 \mu \mathrm{g}$ of the CAAP1 promoter luciferase reporter plasmid and $0.4 \mu \mathrm{g}$ of the MKL1 or/and STAT3 expression plasmid. $0.8 \mu \mathrm{g}$ of pcDNA3.1 vector transfected cells were used as negative control.
After 24 48 hours of transfection, luciferase activity was measured by synergistic TM 4(Bioteck). The transfection efficiency was measured by the general protein concentration of every luciferase assay. All experimental plasmids and different preparations of breast cancer cells performed at least three times, yielding qualitatively similar results. The data in each experiment was three times the mean \pm standard deviation of the representative experiment.

\section{Result}

\subsection{The CAAP1 Promoter Contained Pivotal Binding Sites}

Through software target predictions, the CAAP1 promoter contains several pivotal sites situated at base pairs 799 to 809 (5'-CCAATTTAAG-3'), 2139 to 2148 (5'-TTCTCAGAA-3').

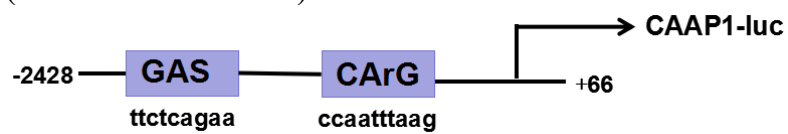

Fig. 1. The CAAP1 promoter contained pivotal binding sites.

\subsection{Construction of luciferase reporter plasmid for CAAP1 promoter gene}

To test whether the PCR of the CAAP1 promoter is correct, we performed agarose gel cataphoresis. In Fig. 2(A), the two bands appeared at $2494 \mathrm{bp}$, which is the CAAP1 gene promoter's PCR product (2428 bp upstream to $66 \mathrm{bp}$ downstream).

We then assimilated these PCR products with restriction enzymes Nhe I and Xho I and ligated these fragments into the pGL3-promoter vector. The recombinant plasmid was then extracted and purified and subjected to agarose gel cataphoresis. The result of agarose gel cataphoresis in Fig. 2(B) is a recombinant plasmid.

To verify the accuracy of these plasmids, we assimilated these plasmids with two clone endonucleases and subjected to agarose gel cataphoresis. As a result in Fig. 2(C), these recombinant plasmids were chopped in two bands. The third lane contains two bands of $5000 \mathrm{bp}$ and $2494 \mathrm{bp}$ representing the pGL3-promoter vector and the CAAP1 gene promoter part, respectively. We then further confirmed the accuracy of the luciferase reporter plasmid for the CAAP1 gene promoter by sequencing. Fig. 2(D) and 2(E) show the results of DNA sequence alignment and the structure of the recombinant plasmid, which indicate that the luciferase reporter plasmid containing the CAAP1 promoter was successful.

\subsection{Luciferase reporter assay}




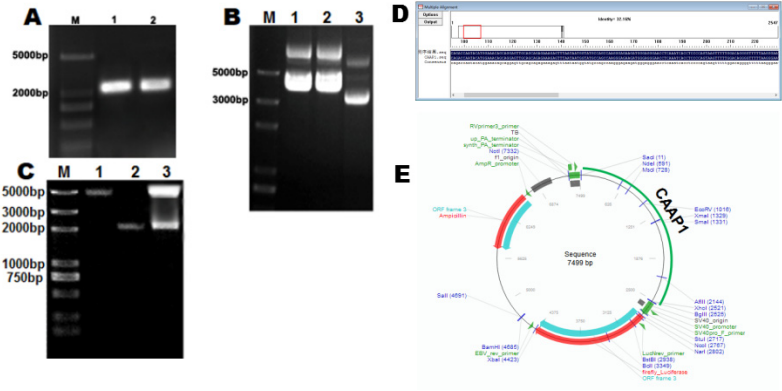

Fig. 2. Construction of luciferase reporter plasmid for CAAP1 promoter gene. (A) Agarose gel cataphoresis pattern of the PCR products of the CAAP1 fragment. (B) Agarose gel cataphoresis pattern of recombinant plasmid. (C) Enzyme digestion to verify the agarose gel cataphoresis pattern of the recombinant plasmid. (D) Comparison of the results of sequencing of recombinant plasmids. (E) Schematic diagram of the structure of the luciferase reporter plasmid containing the CAAP1 promoter.

\subsection{Luciferase Assay}

MCF-7 cells were transfected with MKL1 and STAT3 and CAAP1 promoter for 48 hours and then reported for luciferase reporter gene analysis. As shown in Fig. 3(A), MKL1 showed an obvious effect on enhancing the transcription activity of the CAAP1 promoter compared to the control group. Upregulation of transcriptionally active STAT3 is not particularly evident. Co-transfection of MKL1 and STAT3 can also evidently promote the transcription activity of the CAAP1 promoter. Compared with the control group, there were statistically significant differences in data between groups $(\mathrm{P}<0.001)$. To verify whether CAAP1 is a direct target of MKL1 and STAT3, we carried out site-directed mutagenesis of the luciferase reporter gene containing the wild-type CAAP1 promoter (CAAP1-WT-luc) to construct a CAAP1 promoter mutant (CAAP1-mut-MKL1-luc) with mutant MKL1 binding site, a CAAP1 promoter mutant (CAAP1-mutSTAT3-luc) with mutant STAT3 binding site and a CAAP1 promoter mutant (CAAP1-mut-luc) with two binding sites. These luciferase plasmid vectors were cotransfected into MCF-7 cells with MKL1, STAT3 or pcDNA3.1. The data show that MKL1 or STAT3 significantly increased the activity of the CAAP1-WTluc luciferase plasmid, but had no effect on the activity of the CAAP1-mut-luc luciferase plasmid in Fig. 2(B) (P $<0.001)$. Taken together, these results demonstrate that MKL1 and STAT3 promote CAAP1 expression in MCF7 cells by directly targeting the CAAP 1 promoter.

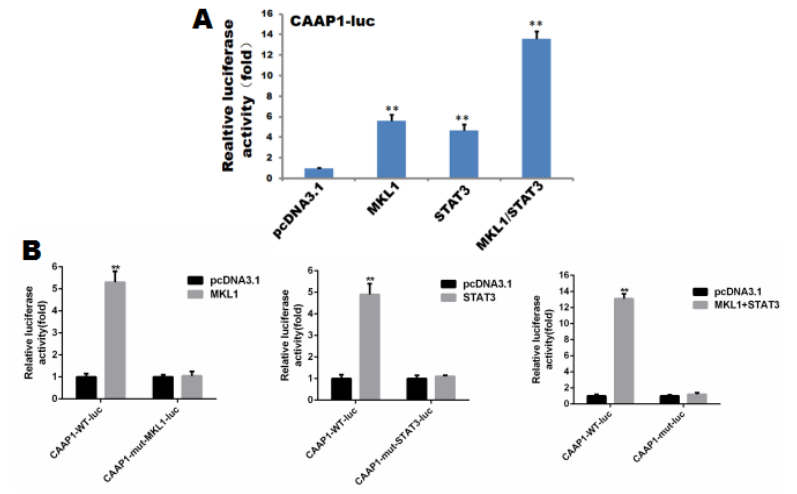

Fig. 3. (A) The relative luciferase activity of CAAP1 by cotransfected with MKL1 and STAT3 and CAAP1 promoter. (B) Relative luciferase activity of CAAP1 after mutation of MKL1 and STAT3 binding sites.

\section{Conclusion}

Breast cancer is the main cause of cancer death in women worldwide, and surgery is still an early primary cures option before cancer metastases develop, and can lead to good patient outcomes[16,17]. However, most patients are usually diagnosed with breast cancer in the late stages of the disease and usually when the tumor has successfully metastasized to other parts of the body[18,19]. Using current diagnostic tools, early detection of breast cancer remains a challenge. Therefore, this requires finding updated, more accurate predictors to help provide better diagnosis and prognosis of breast cancer. CAAP1 is an anti-apoptotic protein that is induced by the activation of cysteine proteases and then cleaves key protein substrates resulting in cell death. Studies have reported that CAAP1 often has mutations or deletions in tumors[7,20]. However, the definitive transcriptional mechanism of the CAAP1 gene is still unclear.

Previous studies have demonstrated that there may be direct protein interactions between MKL1 and STAT3, and they are all closely related to the development of breast cancer[4,17]. Through the target prediction software, we found that there are specific binding sites for MKL1 and STAT3 in the CAAP1 promoter sequence, which indicates MKL1 and STAT3 may directly bind to the promoter of the CAAP1 gene to regulate its expression.

In this study, we successfully constructed the luciferase reporter gene for the CAAP1 promoter. We then tested activation of the CAAP1 promoter in MCF-7 cells by transfecting the CAAP1 promoter with one or both of the expression vectors of MKL1 and STAT3. The results showed that MKL1 can evidently promote the transcription activity of the CAAP1 promoter, and STAT3 can slightly promote the transcription activity of the CAAP1 promoter. Activation can be promoted when co-transfecting expression vectors for MKL1 and STAT3. We then mutated the binding sites of MKL1 and STAT3 on the CAAP1 promoter, and the results showed that MKL1 and STAT3 can promote the transcriptional 
activity of the wild-type CAAP1 promoter, but not the transcriptional activity of the mutant CAAP1 promoter.

Our next step is to demonstrate the binding site of MKL1 and STAT3 to the CAAP1 promoter by CHIP assay in MCF-7 cell line. In addition, more new discoveries of transcriptional regulation can reveal the occurrence and development of breast cancer cells. Our research can also offer some idea and thinking for the cures of breast cancer.

\section{References}

1. R. L. Siegel, K. D. Miller, and A. Jemal, CA: a cancer journal for clinicians 67, 7 (2017).

2. W. Chen, R. Zheng, P. D. Baade, S. Zhang, H. Zeng, F. Bray, A. Jemal, X. Q. Yu, and J. He, Ca A Cancer Journal for Clinicians 66,115 (2016).

3. J. S. Wiest, W. A. Franklin, J. T. Otstot, K. Forbey, M. Varella-Garcia, K. Rao, H. Drabkin, R. Gemmill, S. Ahrent, and D. Sidransky, Cancer research 57, 1 (1997).

4. R. H. Wijdeven, B. Pang, V. D. Z. Sy, X. Qiao, V. Blomen, M. Hoogstraat, E. H. Lips, L. Janssen, L. Wessels, and T. R. Brummelkamp, Cancer research 75, 4176 (2015).

5. H. H. Park, Y. C. Lo, S. C. Lin, L. Wang, J. K. Yang, and $\mathrm{H}$. Wu, Annual Review of Immunology 25, 561 (2007).

6. U. Fischer, R. U. Jänicke, and K. Schulzeosthoff, Cell Death Differentiation 10, 76 (2003).

7. Y. Zhang, E. Johansson, M. L. Miller, R. U. Janicke, D. J. Ferguson, D. Plas, J. Meller, and M. W. Anderson, Plos One 6 (2011).

8. M. Song, F. Fang, X. Dai, L. Yu, M. Fang, and Y. Xu, Febs Letters 591, 934 (2017).

9. M. Kaneda, H. Sakagami, Y. Hida, T. Ohtsuka, N. Satou, Y. Ishibashi, M. Fukuchi, A. Krysiak, M. Ishikawa, and D. Ihara, Scientific Reports 8 (2018).

10. T. Yoshio, T. Morita, M. Tsujii, N. Hayashi, and K. Sobue, Carcinogenesis 31 , 1185 (2010).

11. G. Kerdivel, A. Boudot, D. Habauzit, F. Percevault, F. Demay, F. Pakdel, and G. Flouriot, Molecular \& Cellular Endocrinology 390, 34 (2014).

12. J. Azare, K. Leslie, H. Alahmadie, W. Gerald, P. H. Weinreb, S. M. Violette, and J. Bromberg, Molecular \& Cellular Biology 27 , 4444 (2007).

13. H. T. Lee, J. Xue, P. C. Chou, A. Zhou, P. Yang, C. A. Conrad, K. D. Aldape, W. Priebe, C. Patterson, and R. Sawaya, Oncotarget 6 , 10016 (2015).

14. W. Y. Kuo, L. Hwu, C. Y. Wu, J. S. Lee, C. W. Chang, and R. S. Liu, Theranostics 7 , 647 (2017).

15. N. Wang, L. Wei, Y. Huang, Y. Wu, M. Su, X. Pang, N. Wang, F. Ji, C. Zhong, and T. Chen, Oncology Reports 37, 1537 (2017).

16. J. M. McDaniel, K. E. Varley, J. Gertz, D. S. Savic, B. S. Roberts, S. K. Bailey, L. A. Shevde, R. C. Ramaker, B. N. Lasseigne, M. K. Kirby, K. M.
Newberry, E. C. Partridge, A. L. Jones, B. Boone, S. E. Levy, P. G. Oliver, K. C. Sexton, W. E. Grizzle, A. Forero, D. J. Buchsbaum, S. J. Cooper, and R. M. Myers, Oncotarget 8 , 8226 (2017).

17. X. H. Liao, N. Wang, L. Y. Liu, L. Zheng, W. J. Xing, D. W. Zhao, X. G. Sun, P. Hu, J. Dong, and T. C. Zhang, Cellular Signalling 26, 2370 (2014).

18. Y. Xiang, X. H. Liao, C. X. Yu, A. Yao, H. Qin, J. P. Li, P. Hu, H. Li, W. Guo, and C. J. Gu, Experimental Cell Research 357 (2017).

19. X. H. Zhang, M. Giuliano, M. V. Trivedi, R. Schiff, and C. K. Osborne, Clinical Cancer Research An Official Journal of the American Association for Cancer Research 19, 6389 (2013).

20. G. A. Colditz and K. Bohlke, Ca A Cancer Journal for Clinicians 64,186 (2014). 